thebmj

\title{
Editorials
}

\section{Management of malaria and other severe infections in rural Africa and Asia}

BMJ 2010; 340 doi: http://dx.doi.org/10.1136/bmj.c1527 (Published 17 March 2010) Cite this as: BMJ 2010;340:c1527

\author{
Christopher J M Whitty, professor ${ }^{1}$, Toby Leslie, technical director ${ }^{2}$, Clare I R Chandler, lecturer ${ }^{1}$, \\ Sarah G Staedke, senior lecturer ${ }^{3}$ \\ ${ }^{1}$ Department of Infectious and Tropical Disease, London School of Hygiene and Tropical Medicine, London WC1B 7HT \\ ${ }^{2}$ Health Protection and Research Organisation, Street 10, Taimani Road, Kabul, Afghanistan \\ ${ }^{3}$ LSHTM and MU-UCSF Research Collaboration/Uganda Malaria Surveillance Project, Mulago Hospital, PO Box 7475, \\ Kampala, Uganda
}

christopher.whitty@Ishtm.ac.uk

Delays to treatment cost lives and quick fixes are not the answer

The winner of the Research Paper of the Year category in the 2010 BMJ Group Awards was a paper that described the use of pre-referral rectal artesunate (a rapidly effective antimalarial) in patients with severe malaria.1 This remarkable study conducted in Bangladesh, Ghana, and Tanzania randomised 17826 patients with suspected malaria in rural areas to rectal artesunate or placebo before referral to a health facility. Although mortality did not differ between groups, the composite of death and disability was significantly reduced in people who received artesunate, in an analysis that was restricted to those with confirmed malaria. The effect was largely limited to participants who were delayed for more than six hours before arriving at a health facility, in whom the risk ratio was 0.49 (95\% confidence interval 0.32 to 0.77 ). The findings suggest that in patients with severe malaria in whom treatment is likely to be delayed, prereferral treatment with rectal artesunate could reduce the risk of mortality and disability. 1

The study was an outstanding logistical feat and clearly confirmed the principle that treatment of severe malaria in remote areas can reduce morbidity and mortality. The paper has generated much debate since publication about its methods and implications for practice, and it has raised ethical concerns.2 3 It identified important differences between Asia and Africa, and between children and adults.

Three challenging clinical questions arising from the study do not currently have clear answers.

Firstly, can the approach of giving rectal antimalarials in remote areas where malaria is endemic be operationalised? It is currently unclear if giving rectal artesunate (or equivalent effective antimalarials4) can be made to work outside a trial setting, but the findings of this study suggest that we should try. As with many interventions, deprived communities in rural areas some distance from formal health care have the most to gain but are also those most difficult to reach. It would be inappropriate to set up a parallel 
system only to deliver rectal antimalarial, which should be delivered through existing community based systems providing care. It cannot be assumed that improving care in the community will lead to reduced mortality at a population level, and this would have to be tested.5 The cost of delivering rectal antimalarials for pre-referral use must be weighed against the cost of improving speed of access to good quality health care.

Secondly, how can we enhance the rapid referral of severely sick children and adults to health facilities? The need for rapid referral extends beyond malaria. Delivering rectal artesunate is not a substitute for formal care, but a way to reduce the effect of delayed treatment in cases of severe malaria, and the sooner correct treatment is started the lower the risk of death. The study adds to the overwhelming evidence that delays in treating severe infections kill, and it highlights differences between Asia and Africa. Barriers to rapid referral include inadequate information and cultural beliefs about illness, as well as factors such as transport, distance, direct and indirect costs, relationships within households and between carers and professionals, and patients' or carers' impressions of quality of care at health facilities.6 78 The successful management of illness in the community is usually dependent on functioning health systems. Dealing with any one barrier to referral in isolation will therefore have limited impact without changes to the health system as a whole.

Thirdly, what should we do for patients who have severe febrile disease but do not have malaria? How best to manage severe non-malarial illness is complex. In many areas of Africa and Asia where malaria is endemic, it is common to treat most severe febrile illnesses as if they were malaria.9 Malaria is, however, only one severe infection that may cause mortality. In some areas of Africa and Asia the proportion of severe illness attributable to malaria is decreasing,10 and the relative importance of non-malarial causes of illness is therefore increasing. Alternative causes vary by geographical area, age, and HIV prevalence, but bacterial infections make up a substantial proportion of treatable causes in many settings.11 One approach is to improve diagnosis; the World Health Organization now recommends parasitological testing before antimalarial treatment, and this is a major shift in case management. Rapid diagnostic testing to guide treatment is gaining momentum in remote areas, although introducing tests does not in itself immediately solve the problem of misdiagnosis because clinicians' prescribing behaviour is complex and tests are only one element of this.12 An alternative response is to use syndromic treatment, which combines an antibiotic with an antimalarial in probable severe infection, but the impact of this approach has yet to be tested, and it is not clear which antibiotic is best.

The award winning study clearly shows that substantial delays in obtaining treatment have serious effects on seriously ill patients in Africa and Asia even when effective antimalarial drugs are available. The failure of healthcare systems to deliver effective treatment early contributes substantially to avoidable mortality and morbidity. Fixing this system failure will not be easy or quick, but it must be a priority.

\section{Notes}

Cite this as: BMJ 2010;340:c1527

\section{Footnotes}

- Competing interests: All authors have completed the Unified Competing Interest form at www.icmje.org/coi disclosure.pdf (available on request from the corresponding author) and declare:

(1) No financial support for the submitted work from anyone other than their employer; (2) No 
financial relationships with commercial entities that might have an interest in the submitted work; (3) No spouses, partners, or children with relationships with commercial entities that might have an interest in the submitted work; (4) No non-financial interests that may be relevant to the submitted work.

- Provenance and peer review: Commissioned; not externally peer reviewed.

\section{References}

1. Gomes MF, Faiz MA, Gyapong JO, Warsame M, Agbenyega T, Babiker A, et al. Pre-referral rectal artesunate to prevent death and disability in severe malaria: a placebo-controlled trial. Lancet 2009;373:557-66.

2. Kitua A, Folb P, Warsame M, Binka F, Faiz A, Ribeiro I, et al. The use of placebo in a trial of rectal artesunate as initial treatment for severe malaria patients en route to referral clinics: ethical issues. $J$ Med Ethics2010;36:116-20.

3. Simba DO, Warsame M, Kimbute O, Kakoko D, Petzold M, Tomson G, et al. Factors influencing adherence to referral advice following pre-referral treatment with artesunate suppositories in children in rural Tanzania. Trop Med Int Health2009;14:775-83.

4. Aceng JR, Byarugaba JS, Tumwine JK. Rectal artemether versus intravenous quinine for the treatment of cerebral malaria in children in Uganda: randomised clinical trial. BMJ2005;330:334.

5. Arifeen SE, Hoque DM, Akter T, Rahman M, Hoque ME, Begum K, et al. Effect of the integrated management of childhood illness strategy on childhood mortality and nutrition in a rural area in Bangladesh: a cluster randomised trial. Lancet 2009;374:393-403.

6. Mogensen HO. Finding a path through the health unit: practical experience of Ugandan patients. Med Anthropol 2005;24:209-36.

7. Ansah EK, Narh-Bana S, Asiamah S, Dzordzordzi V, Biantey K, Dickson K, et al. Effect of removing direct payment for health care on utilisation and health outcomes in Ghanaian children: a randomised controlled trial. PLoS Med 2009;6:e1000007.

8. Obrist B, Iteba N, Lengeler C, Makemba A, Mshana C, Nathan R, et al. Access to health care in contexts of livelihood insecurity: a framework for analysis and action. PLoS Med2007;4:1584-8.

9. Reyburn H, Mbatia R, Drakeley C, Carneiro I, Mwakasungula E, Mwerinde O, et al. Overdiagnosis of malaria in patients with severe febrile illness in Tanzania: a prospective study. BMJ2004;329:1212.

10. Ceesay SJ, Casals-Pascual C, Erskine J, Anya SE, Duah NO, Fulford AJ, et al. Changes in malaria indices between 1999 and 2007 in the Gambia: a retrospective analysis. Lancet2008;372:1545-54.

11. Berkley JA, Maitland K, Mwangi I, Ngetsa C, Mwarumba S, Lowe BS, et al. Use of clinical syndromes to target antibiotic prescribing in seriously ill children in malaria endemic area: observational study. BMJ 2005;330:995.

12. Ansah EK, Narh-Bana S, Epokor M, Akanpigbiam S, Quartey AA, Gyapong J, et al. Rapid testing for malaria in settings where microscopy is available and peripheral clinics where only presumptive treatment is available: a randomised controlled trial in Ghana. BMJ2010;340:c930. 